\title{
Effects of wall roughness on turbulent junction flow characteristics
}

\author{
Nikolaos Apsilidis · Panayiotis Diplas · Clinton L. Dancey · Polydefkis Bouratsis
}

Received: 20 May 2015 / Revised: 29 October 2015 / Accepted: 26 November 2015 / Published online: 26 December 2015 (C) Springer-Verlag 2015

\begin{abstract}
Global measurements of turbulent flows at wallcylinder junctions are employed to quantify the effects of wall roughness on the behavior of the horseshoe vortex system (HVS). Two laboratory setups were considered: one with an impermeable smooth wall and a second characterized by a porous hydraulically-rough bed. The measurements were obtained using planar Particle Image Velocimetry. Time-averaged flow topology, turbulence statistics, and instantaneous fields associated with the streamwise and wall-normal velocity components are emphasized. Proper Orthogonal Decomposition (POD) is also applied on the velocity signals to probe into the characteristics of the energetic flow structures. For the Reynolds numbers studied here and the specific differences in the roughness geometry of the bed, a clear trend for increase in flow incoherence due to the rough wall is documented. It is also demonstrated that, in the presence of roughness, vorticity and turbulence spread more evenly throughout the junction. On the other hand, qualitative and quantitative agreement between the smooth and rough bed tests is found in the structure of the downflow and the near-wall jet opposing the bulk flow. The efficiency of POD in analyzing turbulent junction flows is justified based on its results and metrics of modal energy distribution. POD verified in an objective way the role of integral components of the HVS dynamics such as the vortices comprising the system and their interplay with the wall. The decomposition furnishes new evidence about energetic structures that were not captured with the other data analysis
\end{abstract}

N. Apsilidis · C. L. Dancey · P. Bouratsis ( $\square)$

Baker Environmental Hydraulics Laboratory, Virginia Tech, Blacksburg, Virginia 24061, USA

E-mail: napsilid@vt.edu

P. Diplas $(\varangle)$

Civil and Environmental Engineering Department, Lehigh University, Bethlehem, Pennsylvania 18015, USA methodologies. It also confirms the aperiodic behavior of the HVS for the investigated Reynolds numbers.

Keywords Junction flows $\cdot$ Horseshoe vortex $\cdot$ Particle Image Velocimetry

\section{Introduction}

Wall-body junctures are integral components of systems encountered in a number of engineering fields such as aerodynamics, turbomachinery, electronics, and infrastructure hydraulics. Understanding the transport phenomena in these regions is of paramount importance for design, construction, and maintenance purposes. The dynamics of transport processes largely depends on the physics of the flows at the junction. Due to the geometry, the incoming flow undergoes structural transformations in the neighborhood of the body. The moving fluid decelerates, separates from the wall, and changes directions. This activity gives birth to organized and self-sustained flow patterns, which comprise the so-called horseshoe vortex system (HVS).

The behavior of the HVS over a smooth wall has received significant research attention. The reader is referred to the review of Simpson (2001) for a detailed presentation of the state of knowledge on junction flows until the late 90's. This work summarized the effects of factors related to flow and geometry characteristics and elaborated on the difficulties in modeling the unsteady behavior of the HVS. Subsequent studies capitalized on recent strides in experimental techniques (Westerweel et al. 2013) and computational methods (Sagaut 2006, Spalart 2009). The focus has been on the study of turbulent HVSs. The prevalent topology of such flows consists of two to four vortices of varying sizes (Kirkil and Constantinescu 2009, Paik et al. 2007, Praisner and Smith 2006). Wall-vortex and vortex-vortex interactions increase turbu- 
This is the peer reviewed version of the following article: Apsilidis, N., Diplas, P., Dancey, C.L. et al. Exp Fluids (2016) 57: 12. https://doi.org/10.1007/s00348-015-2098-0, which has been published in final form at link.springer.com. This article may be used for non-commercial purposes in accordance with Springer Terms and Conditions for Use of Self-Archived Versions.

lence intensity and bed shear stresses within the junction region. Such phenomena have been attributed to the quasiorganized activity of the HVS manifested in the form of the characteristic zeroflow and backflow modes [introduced by Devenport and Simpson (1990)]. These flow features appear in a number of model studies (Escauriaza and Sotiropoulos 2011, Gand et al. 2010, Kirkil and Constantinescu 2009, Kirkil et al. 2006, Paik et al. 2007, Praisner and Smith 2006, Sabatino and Smith 2009), hence they constitute an established characteristic of turbulent junction flows. The physics of the eruptive processes governing the response of the nearwall region to vortex motion has been detailed by Doligalski et al. (1994). In their review, they link the concentration of wall-generated vorticity into secondary structures with phenomena such as vortex-induced separation (Peridier et al., $1991 \mathrm{a}, \mathrm{b})$ and the development of singularities in unsteady boundary layers (van Dommelen and Shen, 1980). They also identify two major unresolved issues in the study of turbulent junction flows over smooth walls: 1) the discovery of a scheme capable of predicting the onset of the eruptive events and 2) the extension of research to higher Reynolds numbers. More recent studies have expanded earlier findings by emphasizing the role of smaller flow structures (hairpin vortices) in the destabilization of the HVS and the switching between the dominant flow modes (Paik, Escauriaza and Sotiropoulos 2007). The number and intensity of hairpins appear to increase with Reynolds number (Escauriaza and Sotiropoulos 2011). Finally, evidence has been furnished that links HVS dynamics with phenomena occurring outside the juncture, such as the turbulent bursting in the incoming boundary layer (Sabatino and Smith 2009) and corner separation at the flanks of the body (Gand et al. 2010).

In contrast to the well-documented case of junction flows developing over smooth and impermeable walls, there is a paucity of investigations on the (more realistic) roughbed paradigm. In a joint experimental-computational study, Roulund et al. (2005) used Laser Doppler Velocimetry data to train a numerical code in modeling junction flows under a variety of conditions. One of them was bed roughness, in the form of a layer of crushed stones. Computed results revealed a limited influence of this factor on the HVS, expressed primarily as a mild trend of reduction in the flow recirculation area with the increase of roughness. Some caution should be exercised on the interpretation of these findings, however, because of deficiencies of the experimental technique to conclusively resolve the dynamics of the HVS. Ahmed and Rajaratnam (1998) measured increased shear stresses at the junction of a body with a wall made of sand glued on a wooden board. They also found that the footprint of high shear was increased compared to the benchmark, smooth bed case. The elevated uncertainty in the velocity measurements (obtained with a Prandtl tube) precluded the extraction of concrete conclusions about the impact of bed roughness on the junction flow field. Tsikata and Tachie (2013) studied the combined effects of roughness and adverse pressure gradient on a turbulent boundary layer. Although the adverse pressure gradient was not the result of flow blockage from a surfacemounted obstacle (instead, a diverging section of the channel was employed) the results could still be relevant to junction flows. In particular, it was demonstrated that the structure of the boundary layer flow was altered both near and away from the wall. Furthermore, increased turbulence kinetic energies (TKE) and Reynolds stresses were attributed to the rough wall and the pressure field.

The objective of this investigation is to examine the effects of a hydraulically rough and porous bed on the physics of junction flows. Particular emphasis was placed on the behavior of the large-scale, energetic flow structures comprising the HVS. This was pursued experimentally through comparisons of Particle Image Velocimetry (PIV) data. Two wall-cylinder junction setups were considered. The first one consisted of a hydraulically smooth, impermeable bed and is referred as the SB test. For the second setup, gravel grains made for a hydraulically rough, irregular, porous, and immobile bed surface. This experiment will be mentioned from now on as the RB test.

The paper is organized as follows. First, the experimental investigation is described in detail. Next, the mechanics of Particle Image Velocimetry data analysis and post-processing based on Proper Orthogonal Decomposition are presented. The focus is then shifted to the results, where time-averaged flow and turbulence statistics are discussed. This is followed by the analysis of the instantaneous velocity fields. Subsequently, the most energetic POD modes and their contribution to the phenomenon are examined. Finally, an outline of the major findings of this study concludes the paper.

\section{Experimental setup}

\subsection{Facilities and physical models}

Experiments were carried out at Virginia Tech and more specifically at the premises of the Advanced Experimental Thermofluid Research Laboratory and the Baker Environmental Hydraulics Laboratory. For the SB test, a water tunnel with a test section of $1.81 \mathrm{~m}$ (length) $\times 0.61 \mathrm{~m}$ (width) $\times 0.61$ $\mathrm{m}$ (height) was used. The RB experiment took place in a recirculating flume with corresponding dimensions of $15.00 \mathrm{~m}$ $\times 1.20 \mathrm{~m} \times 0.40 \mathrm{~m}$. A $15-\mathrm{cm}$ deep layer of crushed limestone with median particle size diameter of $d_{50}=3.6 \mathrm{~mm}$ comprised the channel's bed. The sediment size was selected within the context of a larger experimental design including clear-water scour tests. However, the length of the flume and the roughness of the bed material allowed for the full development of the free-stream flow, without any sediment bed erosion. These are the conditions reported in this investigation. On the 


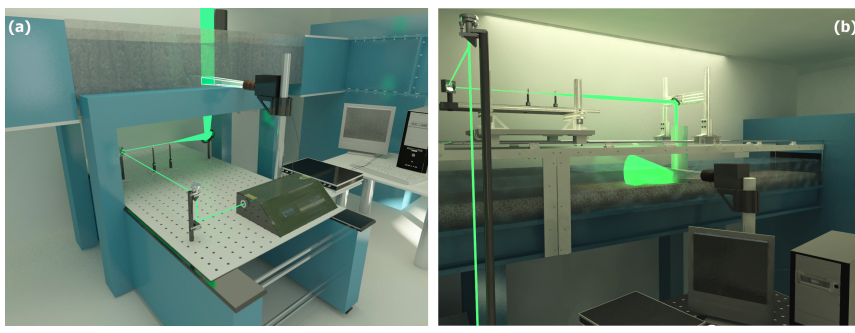

Fig. 1 Physical model and basic components of the non-intrusive PIV setup for: (a) smooth and (b) rough bed tests. Water flows from left to right

other hand, fully-developed flow could not be produced in the water tunnel with the smooth wall. To compensate for this discrepancy, a sand strip comprised of distributed roughness elements of $1 \mathrm{~mm}$ was installed at the inlet of the test section. In this way, it was intended to generate as thick a boundary layer as possible. Past studies (Escauriaza and Sotiropoulos 2011, Koken and Constantinescu 2009, Paik et al. 2007) have advocated that the effect of boundary thickness, $\delta_{99}$, on the dynamics of junction flows is rather limited for the case of relatively thick boundary layers (i.e. those for which $\delta_{99}>0.2 \mathrm{D}$, where $\delta_{99}$ is the boundary layer thickness based on the $99 \%$ of the depth-averaged, free-stream velocity, $U_{0}$, and $D$ is the diameter of the cylinder). The shape factor of the boundary layer developing over the smooth bed was $\theta=1.42$. It is also noted that for the smooth bed test, the diameter of the cylinder was $D=15.2 \mathrm{~cm}$ and the flow depth $H=18.0 \mathrm{~cm}$. The respective numbers for the RB test were $D=8.9 \mathrm{~cm}$ and $H=16.5 \mathrm{~cm}$.

The schematic description of the physical models and the basic components of the PIV systems is presented in Fig. 1. A major goal was to take full advantage of the non-intrusive nature of the PIV technique. For the SB test, the transparent wall conveniently allowed for the delivery of the light from the bottom of the channel (Fig. 1a). On the other hand, the opaque sediment layer of the RB experiment dictated an alternative setup. Two options were considered for the path of laser light through the junction region: a) to penetrate the free surface of the flow and b) to pass through the interior of the cylinder. Solution a) would entail the additional installation of a transparent lid (or a floating window) on the free surface to suppress refraction effects. This configuration could possibly jeopardize the non-intrusive nature of the setup. As shown in Fig. 1(b), it was decided to use a hollow cylinder made of Plexiglass and implement solution b).

In addition to the streamwise-wall-normal plane at the centerline junction, flow measurements were performed at a second station located five cylinder diameters upstream of the cylinder. These data were used to characterize the free-stream flow. Table I summarizes the results as well as other important information related to the conditions of the tests. For the RB case, $U_{0}$ was substantially lower than the critical veloc- ity for the inception of sediment motion. Therefore, and in the absence of suspended sediments, an immobile and porous boundary comprised the roughness layer. For both tests, glass thermometer measurements showed that the water temperature $(T)$ remained practically constant $\left( \pm 0.1^{\circ} C\right)$ throughout the corresponding data acquisition periods. Finally, the levels of flow depth-based Froude $\left(F r_{H}=\frac{U_{0}}{\sqrt{g H}}\right)$, where $g$ is the gravitational acceleration) and cylinder diameter-based Reynolds $\left(\operatorname{Re}_{D}=\frac{U_{0} D}{v}\right)$, where $v$ is the kinematic viscosity of water) numbers for the two tests are reasonably close for practical engineering purposes, allowing for meaningful comparisons (Table 1). Overall, the good agreement between results reported herein and replication runs (one for each test) validated the consistency of experimental setups and methods.

\subsection{Flow diagnostics}

Digital Particle Image Velocimetry was used to probe into the flow physics. Due to limitations in the availability of hardware, different PIV systems were assembled for each case. Measurements for SB were obtained with planar TimeResolved Particle Image Velocimetry (TRPIV). On the other hand, a standard PIV (sPIV) system was employed for the $\mathrm{RB}$ test. The validity of the comparison between results from the two systems is discussed in the Appendix. The next paragraphs highlight similarities and differences between each PIV configuration. Regardless of the characteristics and capabilities of each system, best practices of PIV application [as documented in PIV literature (Adrian and Westerweel 2011, Raffel et al. 2007)] were employed for both cases.

A high-speed CMOS camera and a high-repetition rate Nd: YLF laser were the basic components of the TRPIV system. Their frequency of operation was set to $1 \mathrm{kHz}$, so that images could be recorded according to the continuous-rate mode. The maximum available image size at this frequency was $1,280 \mathrm{pixel} \times 1,040 \mathrm{pixel}$. For the given frequency/image size combination, the 4 GB-buffer of the camera was filled in $3.27 \mathrm{~s}$. A magnification factor of $63.52 \mu \mathrm{m} / \mathrm{pixel}$ balanced the conflicting goals of probing a sizeable field of view at high spatial resolution. This resulted in a maximum error of $1.35 \%$ affecting regions at the outer edges of the images due to deviations of the viewing angle from the optimum of $90^{\circ}$. Hollow glass spheres with an average diameter of 35 $\mu \mathrm{m}$, specific gravity of 1.08 and Stokes number of 0.0051 were used to trace flow trajectories. Homogeneous seeding was obtained by mixing water and particles into the storage tank of the water tunnel. Out-of-plane motion of the seeding particles was mitigated by adjusting the thickness of the laser sheet to $2.0 \pm 0.2 \mathrm{~mm}$. A convex lens with a focal length of $50 \mathrm{~mm}$ and a spherical lens with a focal length of $300 \mathrm{~mm}$ were used for this purpose. The final product was a set of 3270 diffraction-limited images of white particles on a black 
This is the peer reviewed version of the following article: Apsilidis, N., Diplas, P., Dancey, C.L. et al. Exp Fluids (2016) 57: 12. https://doi.org/10.1007/s00348-015-2098-0, which has been published in final form at link.springer.com. This article may be used for non-commercial purposes in accordance with Springer Terms and Conditions for Use of Self-Archived Versions.

Table 1 Major geometric and flow characteristics of experiments

$\begin{array}{ccccccccccc}\text { Test } & U_{0} & D & H & \delta_{99} & \theta & T & F r_{H} & \delta_{99} / D & H / D & \operatorname{Re}_{D} \\ & (\mathrm{~m} / \mathrm{s}) & (\mathrm{m}) & (\mathrm{m}) & (\mathrm{m}) & & \left({ }^{\circ} \mathrm{C}\right) & & & & \\ \text { SB } & 0.486 & 0.089 & 0.180 & 0.077 & 1.42 & 23.5 & 0.36 & 0.87 & 2.02 & 4.7 \times 10^{4} \\ \text { RB } & 0.332 & 0.152 & 0.165 & - & - & 19.7 & 0.26 & 1.08 & 1.08 & 5.0 \times 10^{4}\end{array}$

background, with average particle diameter of $2.3 \pm 0.5$ pixel and density equal to 0.043 particles/pixel ${ }^{2}$.

The sPIV system consisted of a machine vision-type camera equipped with a CCD sensor and a $\mathrm{Nd}$ : Yag laser. The maximum operating frequency of the camera was limited to $15 \mathrm{~Hz}$. Therefore, the frame-straddling mode was selected to ensure optimum particle displacements between successive exposures. The downside of the low sampling rate was counterbalanced by the fact that measurements were statistically independent (time autocorrelation coefficients for velocity signals were consistently less than $15 \%$ throughout the flow domain for the time lag of $133 \mathrm{~ms}$ ) and the data acquisition lasted for $448.8 \mathrm{~s}$. The increased sampling period was due to the capability of the camera to log recorded images directly on the hard drive of the computer. In this fashion, 3,366 image pairs were stored and used to generate an equal number of instantaneous flow fields. The size of each photo was 1,600 pixel $\times 1,200$ pixel. The increased distance between the laser plane and the camera axis for this test (due to the greater width of the flume) entailed several changes in the setup compared to that of the SB test. In particular, the magnification factor was $43.47 \mu \mathrm{m} / \mathrm{pixel}$, which reduced the error due to divergence from the optical axis down to $0.98 \%$. Silver-coated, hollow glass spheres (average diameter: 85 $\mu \mathrm{m}$, specific gravity: 1.1 , Stokes number: 0.0067) were used to increase the intensity of reflected light. The laser beam was manipulated into a $3.0 \pm 0.2 \mathrm{~mm}$ - thick sheet using a convex lens with a focal length of $75 \mathrm{~mm}$ and a spherical lens with a focal length of 1,000 mm. Inside the cylinder, a customdesigned convex mirror with a focal length of $23 \mathrm{~mm}$ was installed to expand the width of the laser sheet. The average particle image diameter was $2.1 \pm 0.5$ pixel and the imaged particle density was 0.035 particles/pixel ${ }^{2}$.

\section{Methods of data analysis}

\subsection{Particle Image Velocimetry}

Despite the differences between TRPIV and sPIV systems, data from both cases were analyzed using common techniques. A number of image preconditioning operations were applied to raw images such as: intensity capping and gradientbased edge detection, masking of stationary objects, and subtraction of time-averaged image (Honkanen and Nobach 2005, Shavit et al. 2007). Instantaneous velocity fields were extracted from successive PIV images using software that implements a Robust Phase Correlation kernel (Eckstein et al. 2008, Eckstein and Vlachos 2009). Multigrid iterative deformable windows (Scarano 2002) were used to probe the turbulent shear flows under investigation. Three passes with two iterations in each and $75 \%$ overlap between the interrogation windows were applied. To mitigate the adverse effects of excessive in-plane particle motion on the spatial correlation signal, discrete window offsetting (Westerweel 1997) was used for the second and third pass. Spurious velocity vectors were identified through a combination of velocity thresholding and Universal Outlier Detection (Westerweel and Scarano 2005). For these unacceptable measurements, "corrected" values were obtained through the application of nearest-neighbor algorithms on an eight-point stencil. Snapshots of instantaneous flow fields comprised of 26,742 velocity vectors for SB and 35,781 data points for RB test. The total number of measurements collected for both experiments was $207,865,660$.

\subsection{Proper Orthogonal Decomposition}

The vast amounts of data typically obtained in PIV experiments are amenable to further analysis using post-processing techniques. One major objective is to extract information about the underlying physical mechanisms by reducing the dimensionality of the data set. This is frequently achieved using modal decomposition (Jolliffe 2002). An a priori selection of the modes (or basis functions) is possible. However, it has been suggested that, for the analysis of turbulent flows, ad hoc functions might be a better alternative (Lumley 1967), because they maximize the projection of the derived empirical modes to the original data set.

Proper Orthogonal Decomposition (POD) is an analyticallyfounded statistical technique, which reduces complex flow fields using energetically optimum basis functions (Berkooz et al. 1993). Considering that the energy content of a moving fluid is proportional to the square of its velocity, POD delivers the smallest number of modes necessary to approximate the original flow field with the smallest possible error (in a least square sense). In other words, the POD-generated basis functions capture more of the kinetic energy of the system than any other decomposition. This makes the technique ideallysuited for the study of flows where large-scale, energetic flow structures dominate. 
The general expression for the decomposition of a discrete velocity signal $\mathrm{u}$, measured in $\mathrm{M}$ realizations varying in the two-dimensional space $\mathbf{x}(\mathrm{x}, \mathrm{y})$ and in time $\mathrm{t}$, is:

$u(\mathbf{x}, t)=\sum_{m=1}^{M} \alpha_{m}(t) \phi_{m}(\mathbf{x})$

Here, $\phi_{m}$ is the $m$-th basis function (or mode) and $\alpha$ is a coefficient. Each mode is a deterministic function in space, whereas the coefficients are stochastic variables in time. Although Eq. 1 expresses essentially a linear operation, there is no restriction for extending its application to non-linear systems. Note also that several types of basis functions could potentially comprise the partial sums of Eq. 1. Two additional conditions need to be satisfied, however, in order Eq. 1 to exclusively express Proper Orthogonal Decomposition (Holmes 2012).

The first condition imposes the orthonormality of the basis functions:

$\iint \phi_{m}(\mathbf{x}) \phi_{n}(\mathbf{x}) d x d y=\delta_{m n}$

where $\delta_{m n}$ stands for Kronecker's delta. In this case, the $m$-th coefficient is uniquely related to the $m$-th mode through the following relationship:

$\alpha_{m}(t)=\iint u(\mathbf{x}, t) \phi_{m}(\mathbf{x}) d x d y$

The second condition ensures the optimality of the decomposition regarding the energy content of the signal. This is achieved when the basis functions are the eigenfunction solutions of the Fredholm integral equation of the second kind:

$\iint R\left(\mathbf{x}, \mathbf{x}^{\prime}\right) \phi_{m}\left(\mathbf{x}^{\prime}\right) d x d y=\lambda_{m} \phi_{m}(\mathbf{x})$

where $\mathrm{R}\left(\mathbf{x}, \mathbf{x}^{\prime}\right)$ is the kernel of spatial cross-correlation and $\lambda$ is the $m$-th eigenvalue of the corresponding basis function. The aggregate of the eigenvalues expresses the total energy of the flow field. The contribution of each mode to the total energy of the system is the relative fraction of its corresponding eigenvalue as described in Eq. 5. It is customary in POD to arrange modes in descending order so that the first modes become the ones that capture the most energetic flow patterns (large-scale, coherent structures).

$\operatorname{EnergyFraction}(E F)_{i}=\frac{\lambda_{i}}{\sum_{j=1}^{M} \lambda_{i}}$

The methodology described so far constitutes the standard version of POD, also known as direct POD. Sirovich(1987) introduced a variation, which is particularly attractive for the decomposition of PIV data. Essentially, he suggested to substitute the spatial cross-correlation kernel $\mathrm{R}\left(\mathbf{x}, \mathbf{x}^{\prime}\right)$ in Eq. 6 with the temporal cross-correlation kernel $\mathrm{C}\left(\mathrm{t}, \mathrm{t}^{\prime}\right)$, defined for the total sampling time (ST) as:

$C\left(t, t^{\prime}\right)=\frac{1}{S T} \iint u(\mathbf{x}, t) u\left(\mathbf{x}, t^{\prime}\right) d x d y$

This change reduces the computational cost, if the total number of instantaneous data snapshots is smaller than that of measurements contained within each field. Hence, the method is known as snapshot POD.

For this study, we applied snapshot POD on the fluctuating signals (time-averaged values subtracted) characterizing the streamwise and wall-normal velocity components for SB and RB tests. The reduced computational cost of this method compared to direct POD allowed us to take advantage of the maximum spatial and temporal resolution of the PIV data.

\section{Results and discussion}

PIV data favor the presentation of results in 2D-maps. Nondimensionalization of the results is performed using characteristic quantities for length $(D)$, time $\left(D / U_{0}\right)$, and velocity $\left(U_{0}\right)$. Since the flow enters the domain from the left, a rectangle is drawn on the right of every map to indicate the location of the cylindrical obstacle. The origin of the coordinate system used here is the intersection of the leading edge of the obstacle with the wall. The horizontal axis ( $\mathrm{x}$ ) is aligned with the direction of the approach flow, $y$-axis is parallel to the cylinder, and $\mathrm{z}$-axis is perpendicular to the measurement plane. The instantaneous velocities in the $\mathrm{x}$ and $\mathrm{y}$ directions are $\mathrm{u}$ and $\mathrm{v}$, respectively.

\subsection{Time-averaged flow and turbulence statistics}

Reynolds decomposition is employed to extract the timeaveraged streamlines from PIV data (Fig. 2). The topology of the SB case is dominated by two foci of closed streamlines organized in an elliptical or spiral pattern (Fig. 2a). In the literature (Simpson 2001), the flow structure on the right is known as the primary vortex (PV) and the one on the left as the secondary vortex (SV). Both vortices gain strength through the same sources, namely the separated boundary layer flow and the reverse flux of fluid that had previously stagnated at the face of the obstacle. The close interaction between the PV and the SV is also expected to be related to their proximity. Note also the higher density of streamlines comprising the $\mathrm{PV}$, which in conjunction with its size, suggests its predominance on the dynamics of HVS. Overall, the two-vortex model presented here bears strong resemblance 
(a)

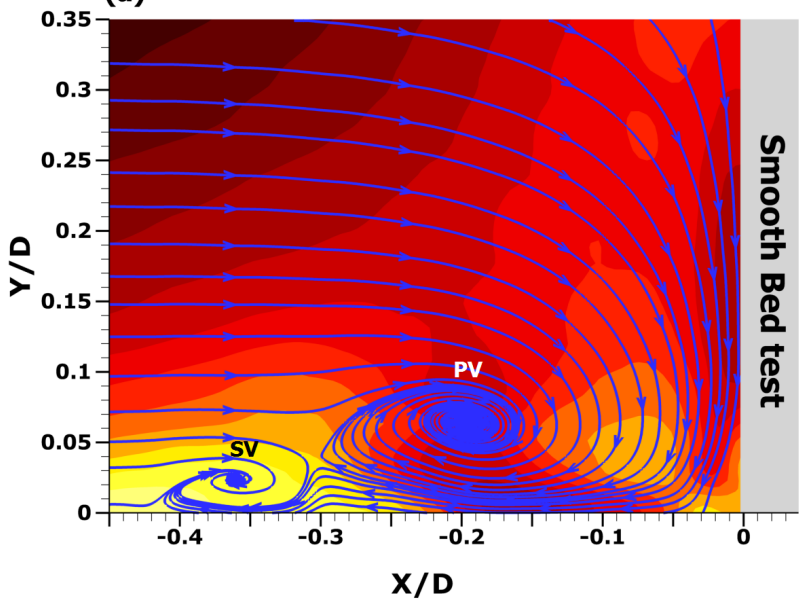

(b)

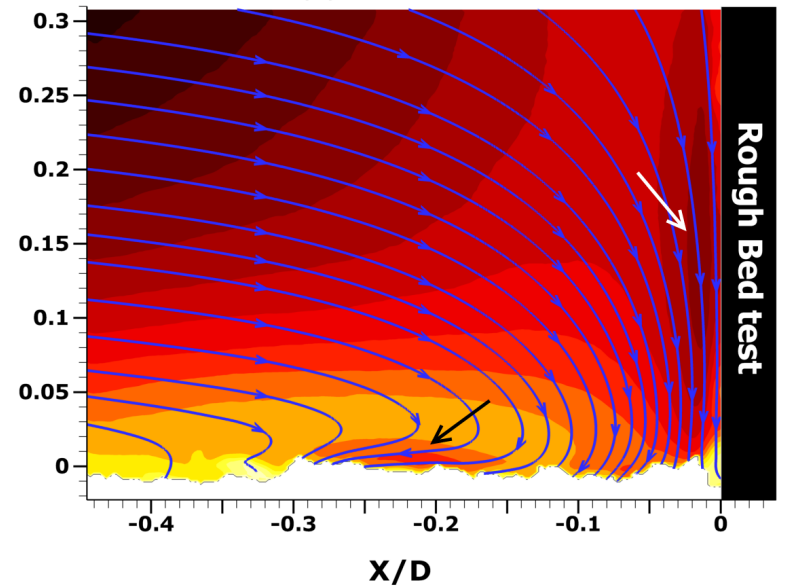

Fig. 2 Time-averaged streamlines drawn on top of flood contours of non-dimensionalized velocity magnitude, $|U|$ for: (a) smooth and (b) rough bed case

to those documented in published studies (Koken and Constantinescu 2009, Paik, Escauriaza and Sotiropoulos 2007, Sabatino and Smith 2009). By contrast, measurements over the rough wall revealed a new topology (Fig. 2b). The absence of any structure manifested with closed streamlines is the most striking characteristic of this flow field. Whereas streamline curvature is evident in the stagnation region, it appears that reversed fluid does not exhibit rotational motion, suggestive of a vortex. It is plausible that time-averaging filters out vortical structures with highly unsteady behavior in time. A closer inspection of the streamlines near the rough wall allows for additional hypotheses. In particular, the path of these streamlines seems to terminate at the wall. This could be suggestive of fluid infiltration into the porous layer. Alternatively, the irregular shape and random orientation of the gravel could deflect fluid elements away from the thin measurement plane. This would entail a continuous wandering of the vortex along the $\mathrm{z}$ (spanwise) direction. Additional work is required to test these hypotheses, because planar PIV precludes flow measurements within the hyporheic zone or outside the laser light plane.

The comparison is now focused on the spatial distribution of the velocity magnitude of the flow. There is a notable similarity regarding the levels and patterns of momentum entering the domain. In both tests, the maximum influx occurs at the upper left corner contributing some $65 \%-70 \%$ of the freestream momentum. The nearly parallel shape of contours indicates a uniform retardation of flow as it approaches the solid boundaries and experiences stronger pressure gradients. Two exceptions to this trend are identified. The first occurs near the face of the cylinder, where masses of downwarddeflected fluid form a jet-like structure that propagates towards the wall (white arrow). The second exception is found close to the horizontal bed, where fluid of high velocity is moving opposite to the direction of the bulk flow (black arrow). Nevertheless, the magnitude and extent of this latter flow pattern differs between the two experiments. For the $\mathrm{RB}$ test, high velocities are limited to a thin strip, which is surrounded by flow of lower velocity (Fig. 2b). On the contrary, for the SB test the reversed jet is wider and becomes part of the lower PV (Fig. 2a).

The rotational content of the junction flows is further investigated through maps of spanwise vorticity (Fig. 3). Vorticity of negative sign is prevalent in both junctions. For the SB case, however, high concentration areas coincide with the locations of the primary and secondary vortex (Fig. 3a). In addition, an elongated patch of negative vorticity emanates from the downflow at the face of the cylinder and stretches all the way to the PV (black arrow). This is indicative of the strong interplay between these two flow components (downflow and primary vortex). Streaks of positive vorticity appear in the immediate vicinity of the wall and the cylinder (maroon arrows). At their ends, the thin streaks are transformed to elliptical or circular blobs of vorticity, which imply the sporadic presence of a tertiary (TV) and a corner vortex (CV).

On the other hand, the rotational characteristics of the RB flow are more subtle (Fig. 3b). The overall levels of negative vorticity are lower compared to those calculated for the SB test. This parameter peaks at a single blob centered on dimensionless $\mathrm{x}$, y coordinates: $(-0.18,0.01)$. The particular location could potentially be home to a vortex. Nevertheless, the origin of such a structure could possibly differ from that of the PV (or SV) as manifested in the SB test. In the presence of roughness (and due to the proximity to the bed) the formation of a vortex could be attributed to flow separation at the edges of sediments that protrude into the flow field. Regardless of the origin of the elevated vorticity, this parameter diffuses gradually and in a relatively uniform fashion 
(a)

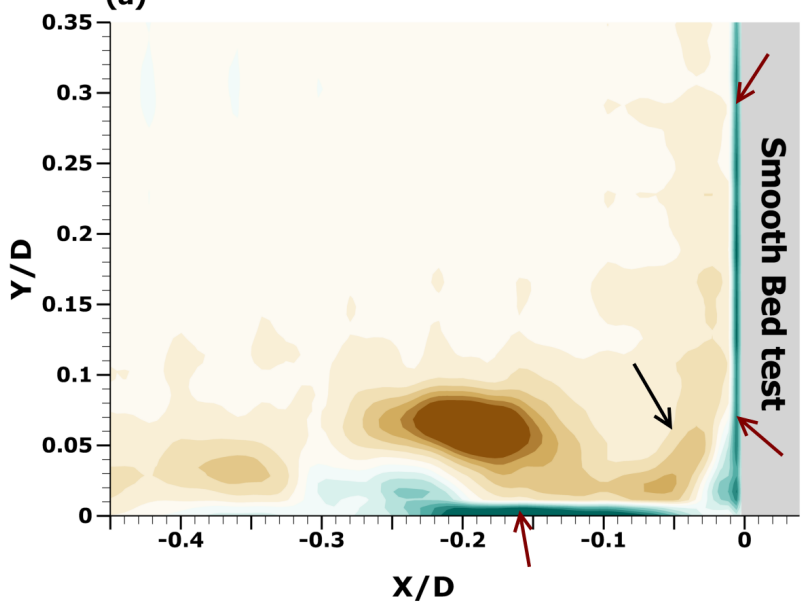

(b)

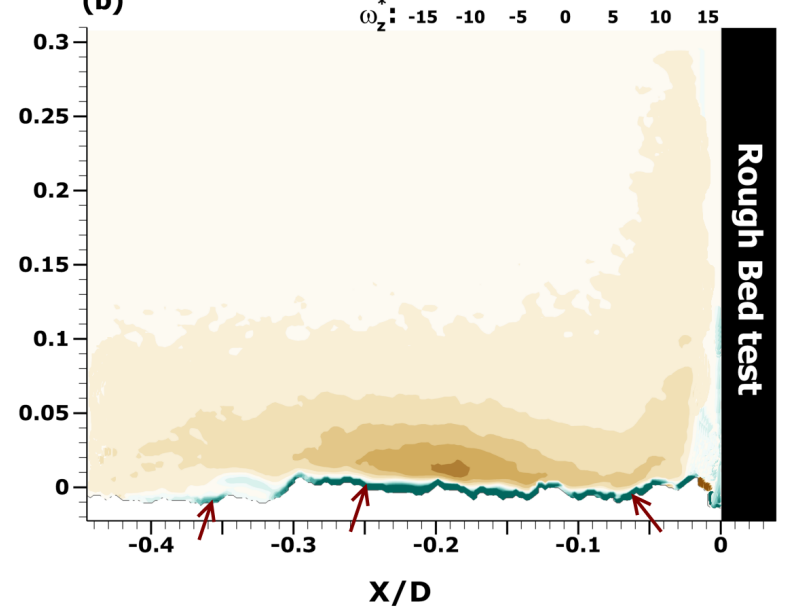

Fig. 3 Spatial distribution of nondimensional spanwise vorticity. (a) SB test, (b) RB test

with height. Eventually, it assumes near-zero values above the average threshold of $\mathrm{Y} / \mathrm{D} \simeq 0.1$. Finally, positive vorticity for the RB case is concentrated exclusively within a thin layer that develops on top of the crests of roughness elements (Fig. 3b, maroon arrows). The time-averaged analysis does not support spatial connections between this layer and other flow components.

Figures 4 and 5 elaborate the turbulent characteristics of the flows under investigation. The evaluation is performed based on the following definitions of the turbulence kinetic energy and intensities:

$T K E=\frac{\left(u^{\prime}\right)^{2}+\left(v^{\prime}\right)^{2}}{2 U_{0}^{2}}$

$T I_{x}=u^{\prime} / U_{0}$

$T I_{y}=v^{\prime} / U_{0}$

where $u^{\prime}$ and $v^{\prime}$ are the rms of the fluctuations of the streamwise and wall-normal velocity components. The turbulence kinetic energy obtains its highest levels within the core and the periphery of the PV (Fig. 4a). Overall, however, TKE retains higher values throughout the RB junction (Fig. 4b) compared to those of the SB case. Differences also exist with respect to specific flow characteristics.

Focusing initially on the SB results, the increased turbulence energy is not confined within the locus of the PV (Fig. 4a). Observe that a band of high TKE emanates from the wall at an oblique angle and extends to the lower part of the PV (black arrow). The energy contained in the band is contributed by the fluctuations of the streamwise velocity component (Fig. 5a). These fluctuations establish the unsteady behavior of the near-well jet of reversed fluid. The combined impact of the unsteadiness of the jet and its interplay with the PV play a pivotal role on the HVS destabilization mechanism proposed based on the episodic extraction of near-wall fluid (Paik et al 2007). Furthermore, increased TKE close to the origin of the coordinate system further supports the hypothesis for the intermittent presence of a $\mathrm{CV}$ (white arrow).

Two prominent flow features dominate the turbulence kinetic energy field of the RB junction: the downflow adjacent to the cylinder and the horizontal, near-wall jet (Fig. 4b). Recall that these were also evident on the velocity map (Fig. $2 \mathrm{~b})$. It is shown here that the structures under consideration are rich in both momentum and turbulence. In support of this statement, turbulence intensity contours in Fig. 5(b) highlight the contribution of each velocity component. Near the cylinder, the turbulent content is expressed almost exclusively through fluctuations in the wall-normal velocity. The dark flood contours versus the blue line contours disclose a difference of nearly an order of magnitude. On the other hand, near the rough wall the disparity is not as pronounced. Whereas the streamwise fluctuations (red line contours) predominate, fluctuations of the wall-normal velocity component remain relevant (gray flood contours). Besides, of interest is the gradual transformation of the turbulence intensity in the central region of the domain, which bridges the two characteristic features elaborated here. The velocity fluctuations vary in a nearly uniform fashion (Fig. 5b), without exhibiting the intricacy and the spatial inhomogeneity documented at the SB junction (Fig. 5a).

At a smaller scale, the effects of the microtopography of the wall are evidenced just upstream of a sediment grain protruding from the average bed level (Fig. 5b, black arrow). A pocket of low turbulence energy and intensity is resolved at the stoss side of this bed irregularity. This "sheltered" area 
(a)

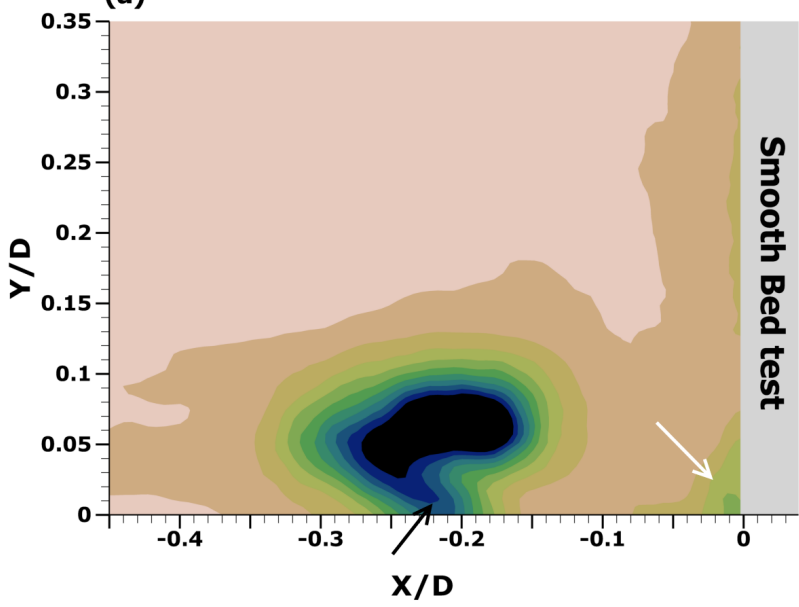

(b)
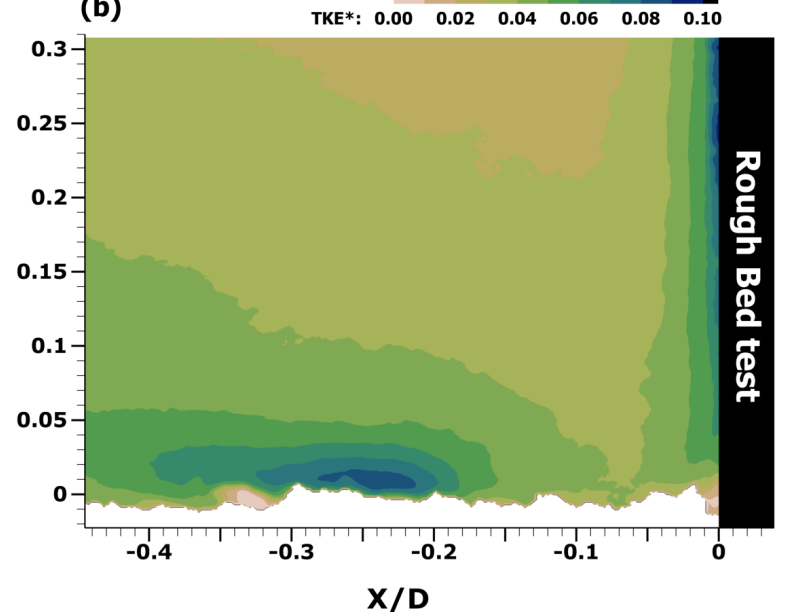

Fig. 4 Non-dimensionalized turbulence kinetic energies (TKE) for: (a) SB test, (b) RB test

(a)

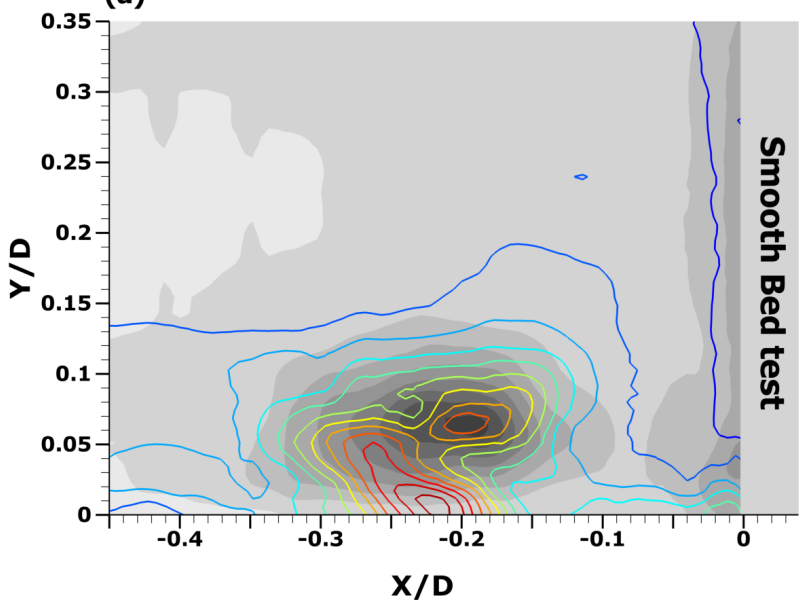

(b)

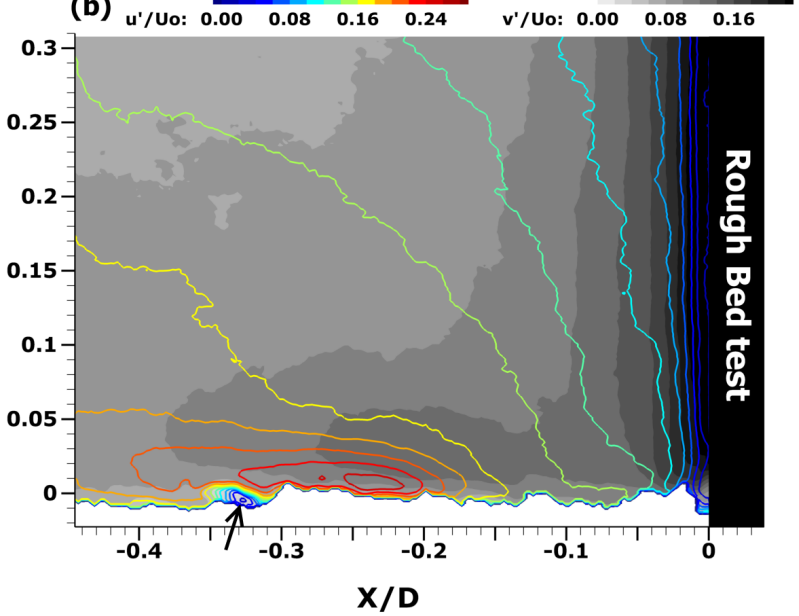

Fig. 5 Contour lines of streamwise turbulence intensity drawn on top of flood contour maps of wall-normal turbulence intensity. (a) SB case, (b) RB case

is also characterized by low levels of vorticity (Fig. 3b) and possibly of flow recirculation (Fig. 2b).

\subsection{Instantaneous velocity fields}

To take full advantage of the information contained within the PIV data, a meticulous, frame-by-frame analysis of the resolved flow fields was performed. The cinematography was constructed using every instantaneous PIV snapshot and using the full spatial resolution of measurements. TRPIV data maximized the benefits from such an analysis. For the sPIV data, the identification of meaningful patterns posed a greater challenge. Nonetheless, it was still possible to shed insights on the physics of junction flows that were not detectable in the time-averaged analysis.
The number of vortices and their interactions defined the dynamics of the HVS for the SB case. Every flow field (frame) comprised of at least one vortex characterized by elevated concentrations of negative vorticity. The maximum number of vortices resolved in the junction region was five. Therefore, the topologies observed on a frame-by-frame basis were more complex compared to their time-averaged representation (Fig. 2a). Although seemingly random at a first glance, the behavior of the HVS was not devoid of organized patterns. For example, the well-known modes for junction flows (zeroflow and backflow) were identified on a number of frames. It was also found that for a significant amount of time the flow characteristics did not comply with those of the aforementioned modes: the strong near-wall jet of reverse flow that extends to the far upstream of the junction (backflow, see Fig. 6a) or the blockage of this jet and its 

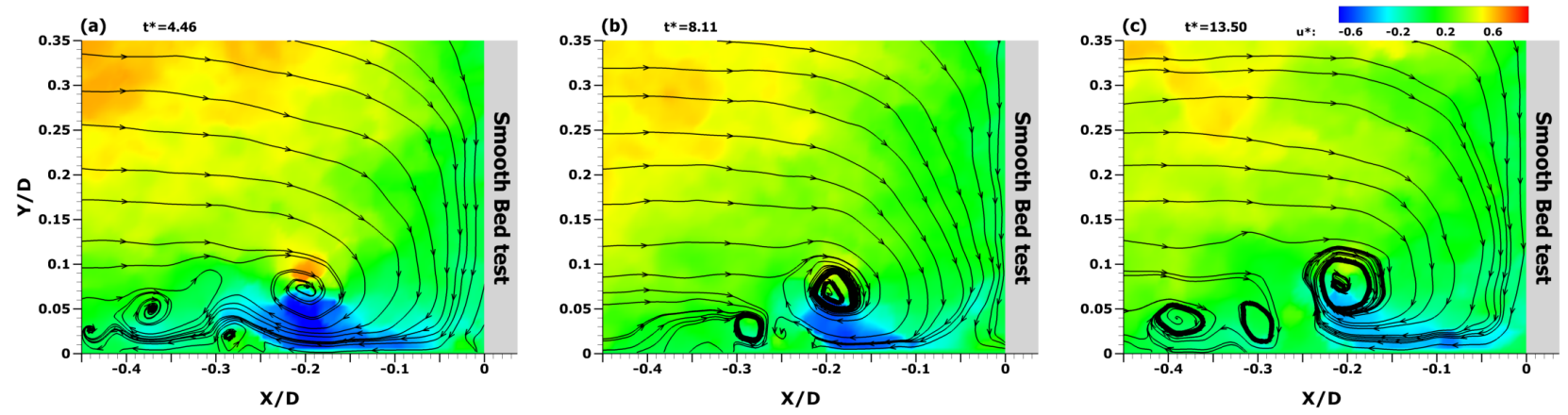

Fig. 6 Illustration of characteristic flow modes for SB test: (a) backflow, (b) intermediate, and (c) zeroflow. Instantaneous streamlines are drawn on top of instantaneous streamwise velocity (nondimensionalized). The dimensionless time of each frame is indicated with $t^{*}$

vertical ejection (zeroflow, shown in Fig. 6c). There were a variety of flow configurations or topologies which were observed to occur besides the back-flow and zero-flow modes, not all of which can be regarded as transitional between these two well-known modes. In other words, direct switching between the zero and back-flow modes does not always occur. One of these is shown in Fig. 6(b). These other flow configurations are intermediate between the clearly identified extreme modes and do include transitional topologies but others which are not necessarily transitional in nature. As these other topologies occur nearly $1 / 3$ of the time, these are termed here the intermediate mode. It is noted that the intermediate mode could include a number of phenomena relevant to the mode-switching mechanism.

Time-resolved analysis revealed additional details about the wall-vortex interactions, which were introduced in the previous section. Figure 7 consists of two episodes indicative of this mechanism. The first one describes the extraction of positive vorticity from the wall, resulting from the complex interplay between the PV, the reverse near-wall jet, and incoming flow that has leapfrogged the SV (Fig. 7a, black arrow). The extracted vorticity does not diffuse into the flow field. Instead, it is captured and recirculates within the TV. Recall that time-averaged analysis furnished scant direct evidence for the existence of this vortex. Figure $7 \mathrm{a}$ also suggests that the high vortical contents of the PV and the reverse nearwall jet are instrumental in shaping the dynamics of a new vortex (TV). This is justified by the absence of a quaternary vortex $(\mathrm{QV})$ upstream of the SV. Although the flow features around PV and SV are topologically similar, the reduced levels of vorticity within the SV and the jet underneath prevent the creation of QV. On the other hand, the second episode of Fig. 7(b) demonstrates that in the absence of secondary vortices, wall-ejected fluid penetrates deeper into the junction flow (maroon arrow). In subsequent frames, these vortical patches will either diffuse or become advected by the outer streamlines of the PV until they join the near-wall jet and start a new cycle of activity.
Fundamentally different were the results of the instantaneous flow analysis for the RB case. Video animations revealed a highly unsteady flow field, which was more difficult to interpret compared to that of the SB experiment. In several frames, there was little evidence of organized flow activity in the form of vortices with properly closed streamlines. Figure $8(a)$ is such an example. It is shown here that the near-wall region is home to small-scale structures (black arrows), which do not exhibit the large-scale behavior of the HVS described in the SB test. Occasionally, separated flow at the junction would reorganize into patterns that are more coherent. The maximum number of vortices detected for the rough HVS system is two (Fig. 8b, maroon arrows). These structures are of similar magnitude in size and coherence. As such, they do not comply with the characteristics of the smooth HVS model, according to which the PV consistently overwhelmed the other vortices in size (Fig. 6, Fig. 7). Finally, two characteristic topologies are reported for the downflow at the face of the cylinder. The first, which occurs in $96 \%$ of time, consists of a downward-deflected jet similar to the one observed in the SB test (Fig. 8b, white arrow). The second topology (present in the remaining $4 \%$ of time) is characterized by small vortices Fig. 8a). These run down the face of the cylinder, rotate in a counterclockwise sense, and disappear from the field of view just as they are about to encounter the rough bed. This pattern was not observed in the SB case, where the corresponding vortices typically propagated all the way to the bed-cylinder junction to form a corner vortex.

The discussion on the dynamics of the junction flow over a rough wall concludes with the description of an organized flow episode. This is illustrated with a sequence of four instantaneous flow field snapshots comprising Fig. 9. The focus is on the larger-scale flow pattern that enters the junction domain from the left bottom at the dimensionless time of $t^{*}=118.64$ (Fig. 9a, black arrow). The development of a single vortex so far upstream was not observed in any frame of the video animations for the SB flow. In the presence of a rough bed, the vortex progressively moves closer to the cylinder, despite the strong near-wall jet propagating in the 

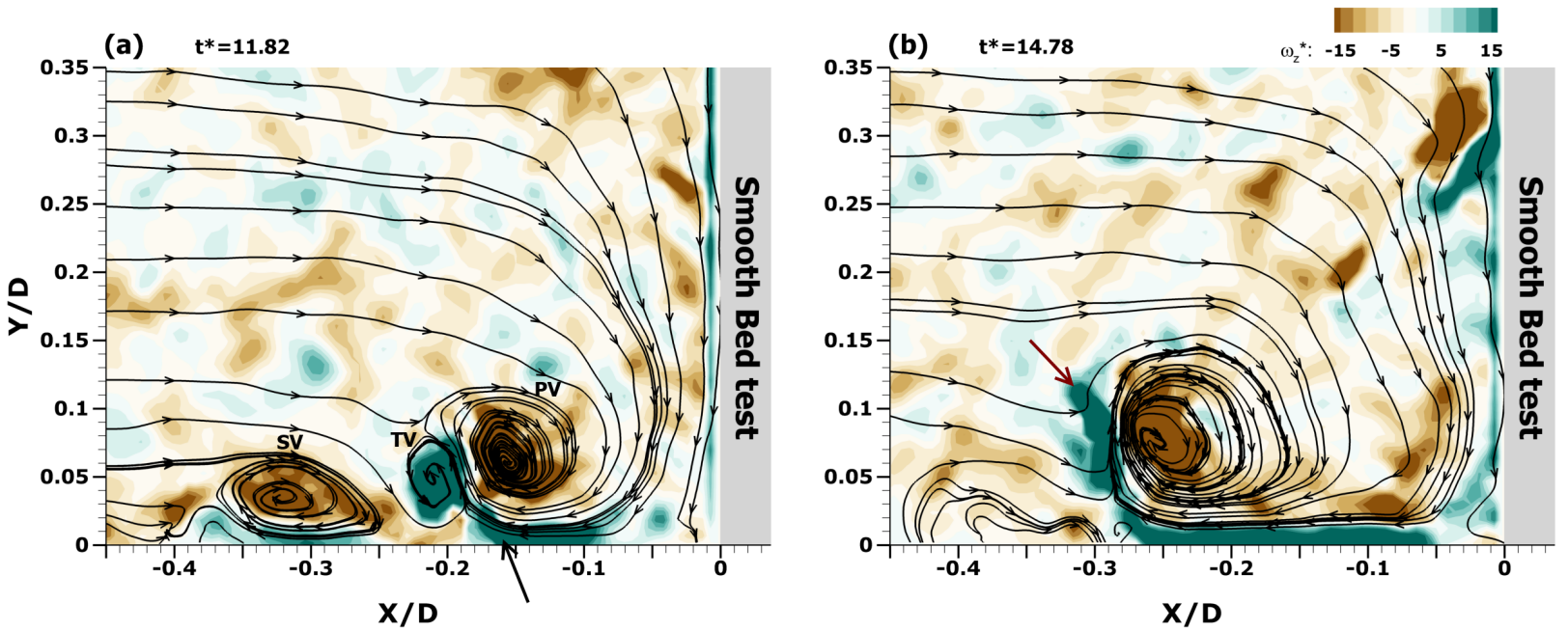

Fig. 7 Instantaneous flow topologies for SB case demonstrating wall-vortex interactions: (a) PV, SV, and TV are present. (b) PV is dominant. Flow streamlines are superimposed on flood contours of the magnitude of nondimensionalized, spanwise vorticity

\section{(a) $\quad t^{*}=25.44$}

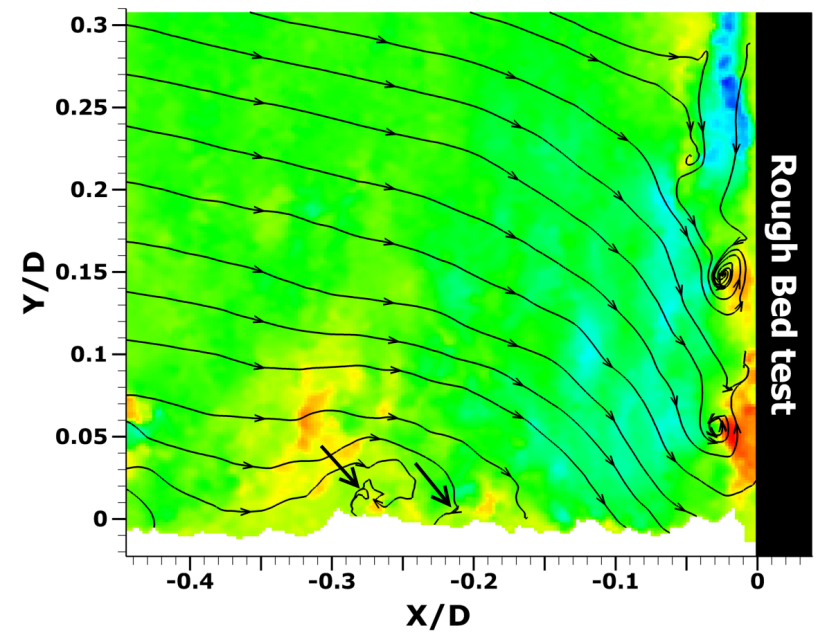

(b)

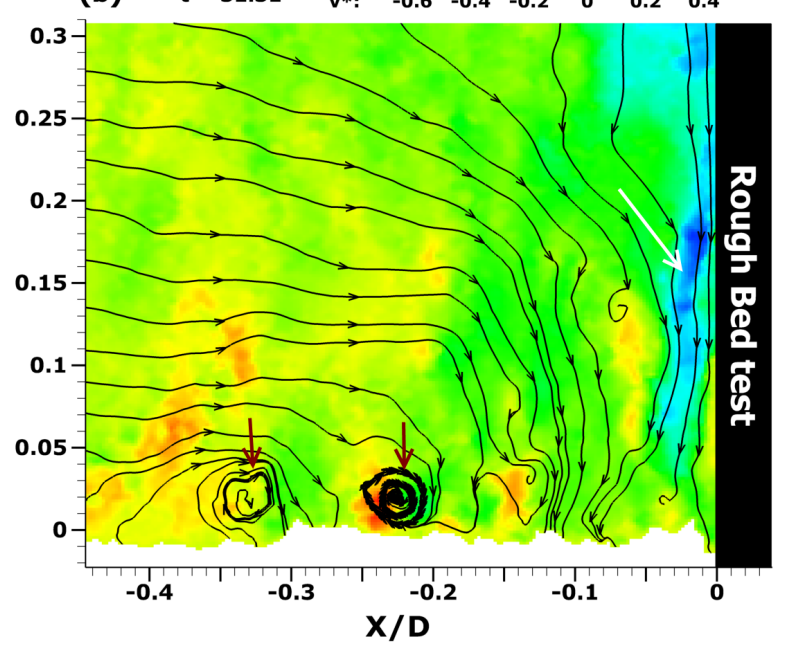

Fig. 8 Characteristic snapshots of the RB flow field: (a) vortices running down the face of the cylinder, (b) vortices developing along the rough wall. Flood contours display instantaneous wall-normal velocity

opposite direction (Fig. 9b, white arrow). It is possible that the propulsion of the vortex is facilitated by the influx of highmomentum fluid occurring at the mid and top of the domain (areas colored in orange and red). The core of the vortex does not remain constant. It is subject to expansion (Fig. 9b) and contraction (Fig. 9c). Eventually, this flow structure is disintegrated at $t^{*}=120.40$ (Fig. 9d). The loss of its coherence coincides with the retreat of high-momentum fluid.

\subsection{POD modes}

The number of POD modes necessary to reconstruct the original signal to a predetermined (though generally arbitrary) degree indicates the level of flow organization. Regular flows, with periodic characteristics in space and time require relatively few modes (low dimensionality). On the other hand, POD yields an increased number of modes for highly unsteady and inhomogeneous flows (high dimensionality). Figure 10 displays the energy content of the POD modes for the SB and RB tests. For both cases, most of the energy is contained in the first two modes. The energy fraction for modes 1 and 2 is $13.98 \%$ and $9.42 \%$ for the SB case. The corresponding energy fractions for the RB test suggest more energetic modes, measuring $28.78 \%$ and $15.24 \%$. Nonetheless, all these values in the low range of values reported in the literature. It is not uncommon for the first few modes of turbulent processes describing other systems to capture more than $80 \%$ of the total energy (Cruz et al. 2005, El Hassan and Meslem 
(a) $t^{*}=118.64$

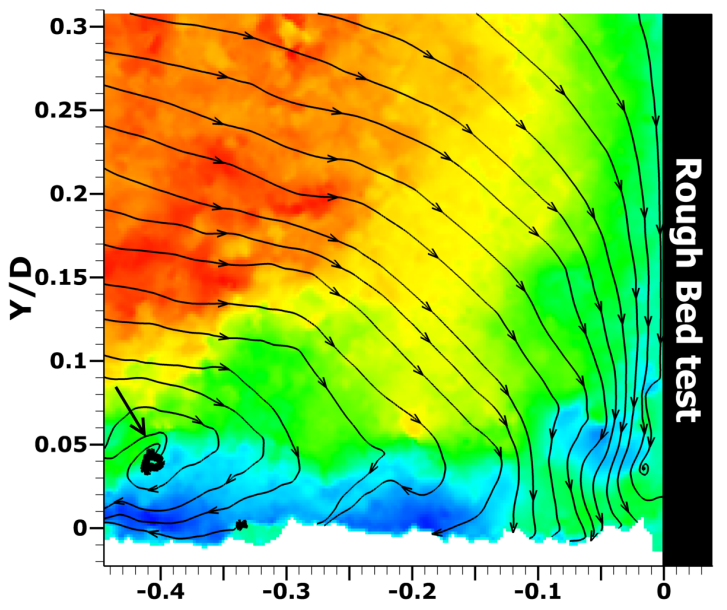

(c) $t^{*}=120.11$

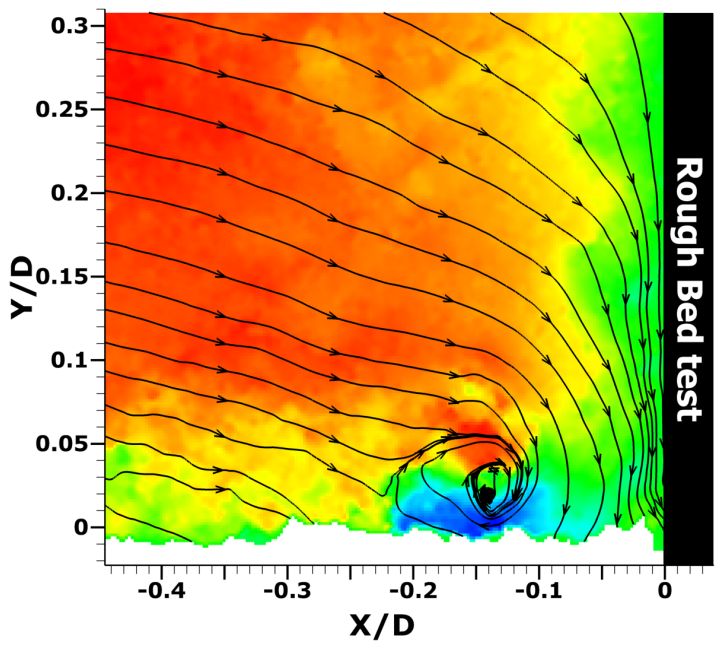

(b)

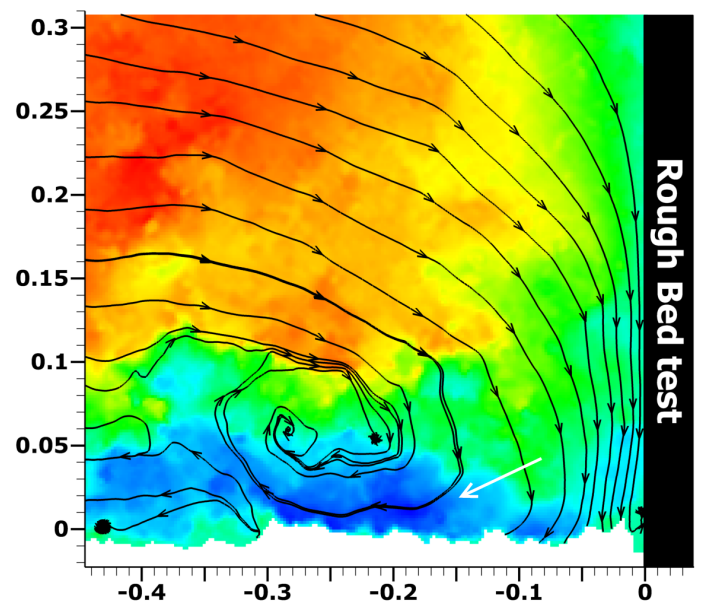

(d) $t^{*}=120.40$

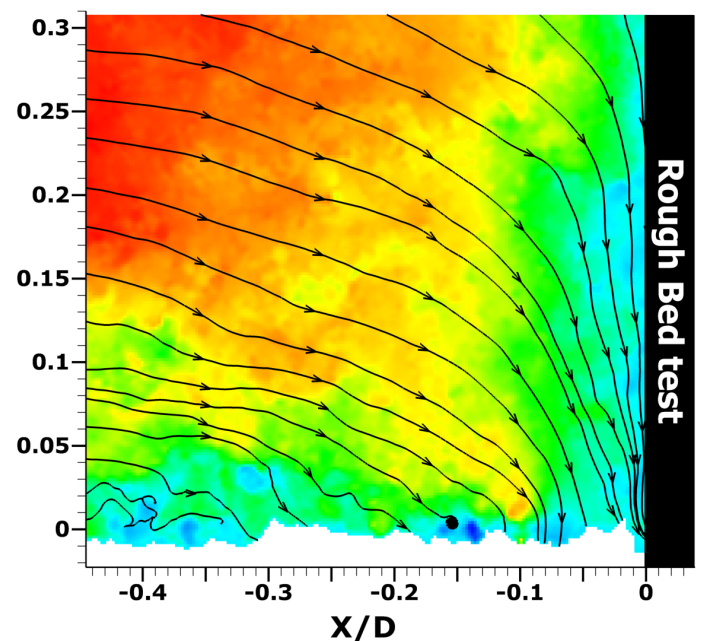

Fig. 9 Sequence of snapshots displaying the unsteady vortex dynamics for the RB test. Background contours are colored with the instantaneous streamwise velocity. The dimensionless timestamp is denoted with $t^{*}$

2010, Nguyen et al. 2010). In our study, it required 10 modes for the SB case and 4 modes for the RB case to capture 50\% of the total kinetic energy. For the level of $90 \%$, the number of modes are 145 and 140 respectively. Due to the elevated energy content of the first two modes, energy convergence for the RB case is faster than that of the SB case for the first 160 modes. At this threshold, the cumulative energies assume nearly identical values and, from this point on, their convergence continues at practically equal rates until 100\% is recovered.

Prior to the analysis of the dominant POD modes, it is reminded that they are projections of the most energetic flow patterns on the spatial domain. The mechanics of POD ascribe a deterministic character to these spatial structures. Therefore, POD modes are not to be interpreted similarly to typical flow fields (4.1). For this reason, they are drawn as continuous lines that are reminiscent to streamlines (pseudostreamlines), but contain no information about the direction of flow motion. Furthermore, the POD modes are superimposed on flood contour maps of their Euclidean norm, which has the following general form for the $m$-th mode:

$\left|\phi_{m}(\mathbf{x})\right|^{2}=\phi_{u m}^{2}(\mathbf{x})+\phi_{v m}^{2}(\mathbf{x})$

Based on the rapid decay of modal energy (Fig. 10), the analysis focuses on the leading four eigenmodes.

Modes 1 through 4 for the SB experiment are shown in Fig. 11. Four structures characterize the most energetic mode (Fig. 11a, A1-A4). Three of them (A2-A4) appear close to the bed and are, logically, related to the HVS. In particular, the two structures closer to the cylinder (A3 and A4) could express the preferable locations of $\mathrm{PV}$ in an energy-wise sense. 
This is the peer reviewed version of the following article: Apsilidis, N., Diplas, P., Dancey, C.L. et al. Exp Fluids (2016) 57: 12. https://doi.org/10.1007/s00348-015-2098-0,

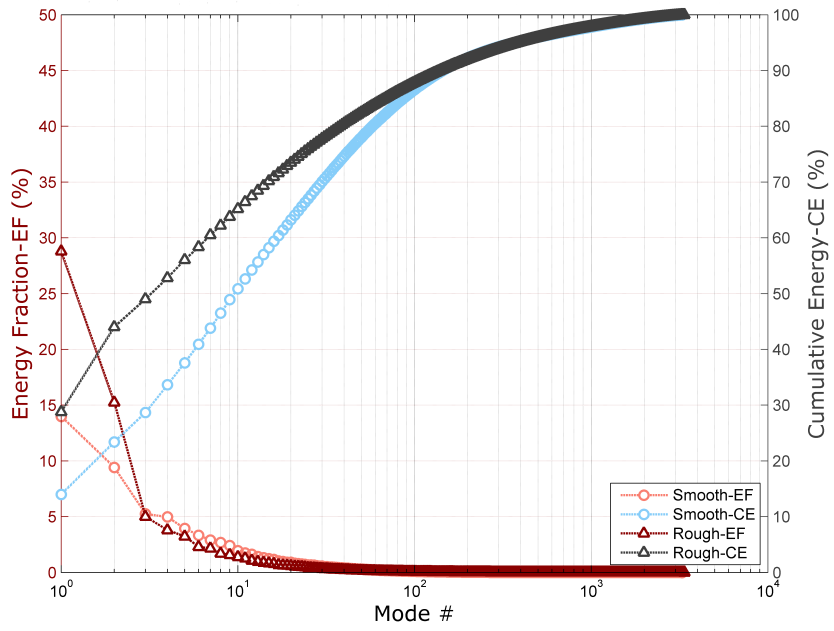

Fig. 10 Energy fraction (EF) and cumulative energy (CE) distribution of the POD modes

The third one (A2), further upstream, manifests in the area of action of SV. The interpretation of structure A1 appearing at the left top of the domain is not straightforward. It is conjectured that it cumulatively conveys the high-energy content of the incoming flow. Of interest are also the areas flooded in green color, where the Euclidean norm peaks. The one with the round shape (white arrow) expresses the kinetic energy of the PV. The second (black arrow) provides additional evidence to support the mechanism discussed in previous sections and consists in the near-wall extraction of fluid resulting from the interaction of the lower part of the PV with the bed. Mode 2 is described with only one characteristic structure representative of a PV topology, B1, which was also observed in several frames of the flow cinematography (Fig. 11b). The Euclidean norm of the $u$ and v POD submodes is again higher at the neighborhood of the HVS, but is characterized by lower concentrations compared to those of mode 1 . This parameter regains high values on the third most energetic decomposition (Fig. 11c). There is a striking similarity between the areas of increased $|\phi|^{2}$ shown here with those of mode 1. Most likely, these express the same flow mechanism and their re-appearance in a subsequent mode underscores the importance of their role. Pseudostreamlines of mode 3 allude to a train of organized structures at the HVS region (C1-C4). Here, for the first time POD resolves a flow structure, $\mathrm{C} 4$, which is candidate for expressing the $\mathrm{CV}$. The last mode examined for the SB test consists of three patterns worth commenting on (Fig. 11d, D1-D3). The ones close to the wall (D1 and D2) most likely represent the same physical processes in the HVS examined in previous modes. In this case, however, they are shifted spatially to the upstream of the junction. Regarding D3 -the larger structure near the cylinder-, it is postulated that together with the adjacent vertical band of high $|\phi|^{2}$ indicate the energetic content of the downflow.
The examination of the corresponding POD modes for the RB test disclosed significant differences compared to the SB experiment (Fig. 12). To begin with, the energy of the first mode is not distributed to any structure reminiscent of those typically found in junction flows (Fig. 12a). Pseudostreamlines are organized in a nearly parallel fashion. In addition, the concentration of the Euclidean norm suggests the higher energy content of the incoming flow (left) as opposed to that of the stagnation region (right). Mode 2, on the other hand, is characterized by the dominant structure b1 centered on $(-0.29,0.12)$. The distance from the rough boundary casts doubts about the connection of this feature with the HVS (Fig. 12b). Besides, a trail of higher concentrations of $|\phi|^{2}$ crosses different areas of the flow field, such as the upper part of the incoming flow, the downflow at the face of the cylinder, and the reversed flow jet near the bed (black arrows). For the latter, an increment in the concentration at a narrow band between $-0.20 \mathrm{D}$ and $-0.32 \mathrm{D}$ complements the time-averaged analysis and establishes the importance of the jet in terms of energy content. This is further justified by the presence of this feature in the third POD mode (Fig. 12c). The role of the two dominant structures shown on this map (c1 and $\mathrm{c} 2$ ), however, is not as clear. In particular, c2 consists of numerous pseudostreamlines that organize around their center. It could be plausible that such a pattern focuses the kinetic energy of fluid in the stagnation region. On the other hand, there is more confidence that the structure $\mathrm{c} 1$ expresses one of the positions assumed by the intermittent vortices of the HVS. Another position is suggested on the map of mode 4 (Fig. 12d). In this case, the energetic structure 2 is located closer to the cylinder. Due to its size, it is most likely to represent the PV (instead of the CV, which is typically smaller). Finally, $\mathrm{d} 2$ - the small-scale structure resolved at $(-0.40,0.12)$ eludes a straightforward interpretation. The same holds for similar structures that increase in number and appear more often as the rank of modes increases.

\section{Conclusions}

Time-averaged flow maps and turbulence statistics provided a first approximation on the effects of wall roughness. The most striking finding was the roughness-induced modulation on the large-scale characteristics of junction flow, manifested with the absence of properly closed vortical structures. The RB flow also experienced extensive diffusion of vorticity, turbulence intensity, and turbulence kinetic energy throughout the domain. On the smaller scales, bed surface irregularities modified the local streamline curvature. This induced pockets of low turbulence (stoss side) alternating with more energetic flow patterns (lee side). Nevertheless, qualitative and quantitative similarities also existed regarding the levels of momentum entering the junction, the prevalent sign of 

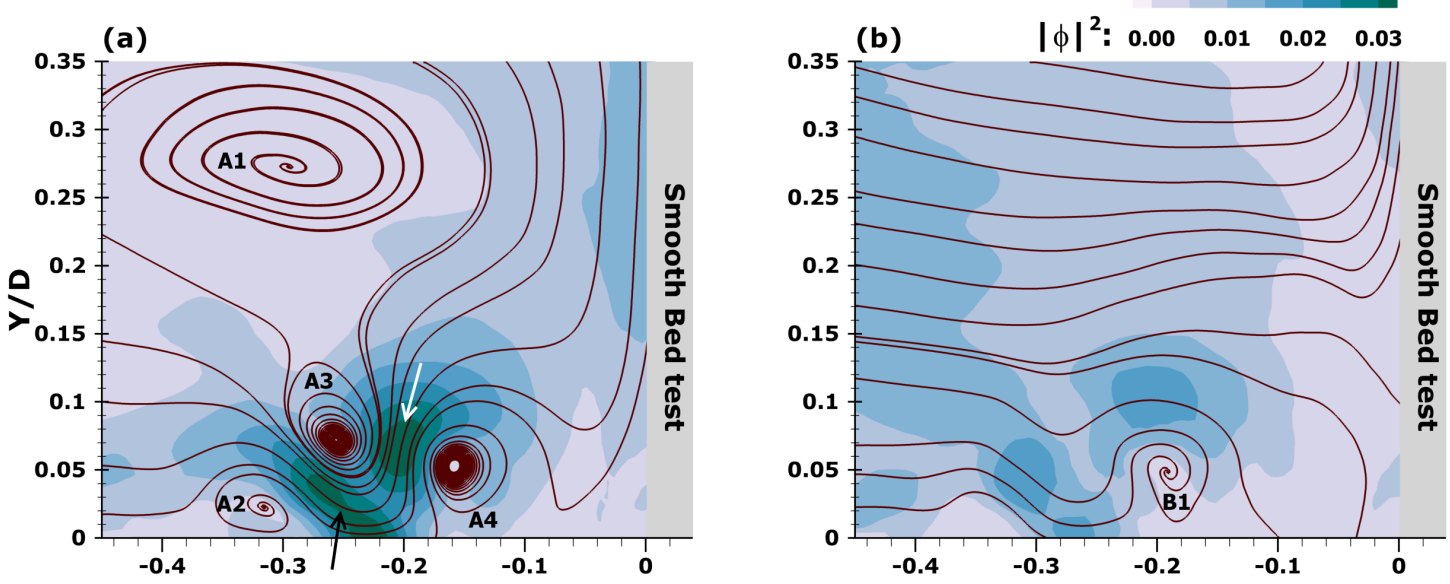

(c)

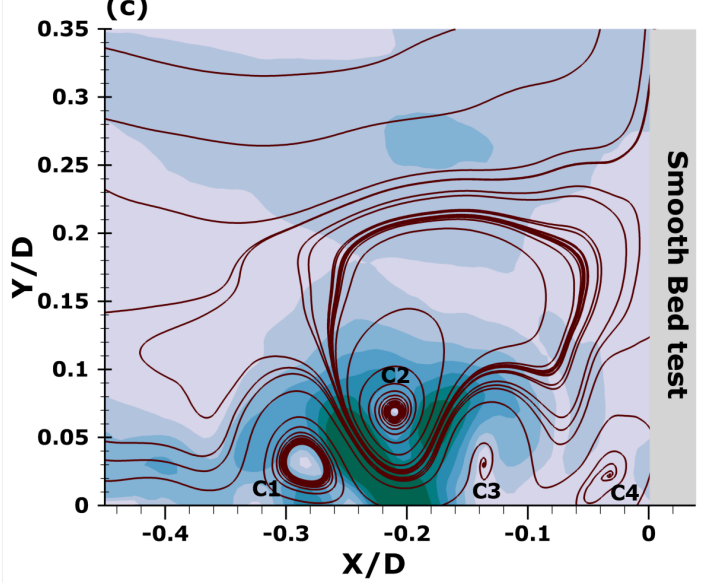

(d)

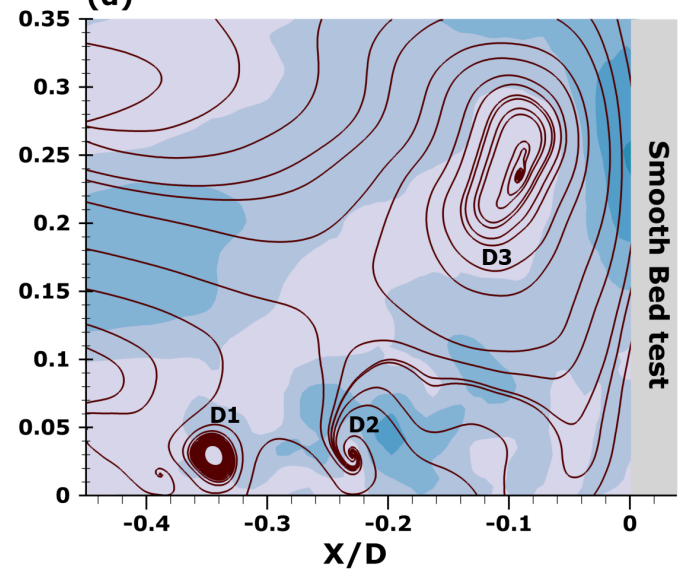

Fig. 11 (a) First, (b) second, (c) third, and (d) fourth POD mode (pseudostreamlines) for the SB test. In the background, flood contour plots map the distribution of the Euclidean norm of the decomposition for the streamwise and wall-normal velocity signals

vorticity for the incoming flow, the reverse near-wall jet, and the downflow.

The instantaneous analysis elucidated the dynamics of two phenomena unique to the SB test: the presence of an intermediate flow mode and the ejection of wall-fluid due to wall-vortex interactions. The well-defined flow topologies resolved in this test enabled the presentation of the unsteady dynamics of the HVS in an organized fashion. On the contrary, the rough wall entailed modifications to the degree of coherence of the flow. The smaller number of vortices, their seemingly erratic behavior, and their focus on new areas of action suggested a radically different HVS model for the RB test compared to that of the SB one. Important discrepancies also characterized the incoming flow: whereas for the latter test a key role for this flow feature was to impart momentum and energy directly on the PV, for the former its main contribution consisted in the advection of the PV towards the cylinder.
Proper Orthogonal Decomposition proved to be a useful tool for the analysis of junction flows. For the SB test, the decomposition confirmed the major flow patterns derived from the time-averaged and time-resolved analyses. It also yielded information about new energetic structures present in the incoming flow and the downflow. Finally, POD rigorously established the wall-vortex interaction mechanism, which manifested with the ejection of near-wall fluid. For the RB data, spatial POD modes mostly revealed structures for which no indication had been provided through methodologies based on Reynolds decomposition. Although the role of these new structures is not always obvious, they are still worth considering within the frames of reduced order modeling. Regarding the mechanics of POD, the concentration of kinetic energy was higher for the leading modes of the RB experiment compared to those of the SB test. Nevertheless, for the relatively high Reynolds numbers investigated here, the energy contribution of these modes is not as great compared to that typically recovered in systems characterized by 
This is the peer reviewed version of the following article: Apsilidis, N., Diplas, P., Dancey, C.L. et al. Exp Fluids (2016) 57: 12. https://doi.org/10.1007/s00348-015-2098-0, which has been published in final form at link.springer.com. This article may be used for non-commercial purposes in accordance with Springer Terms and Conditions

(a)

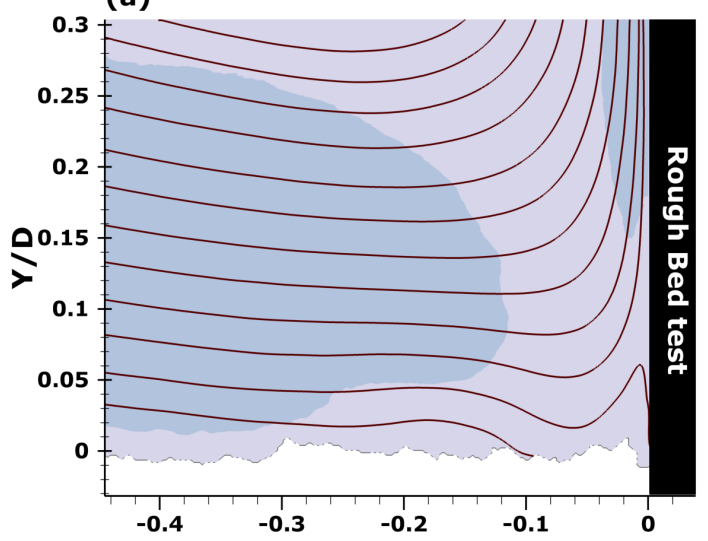

(c)

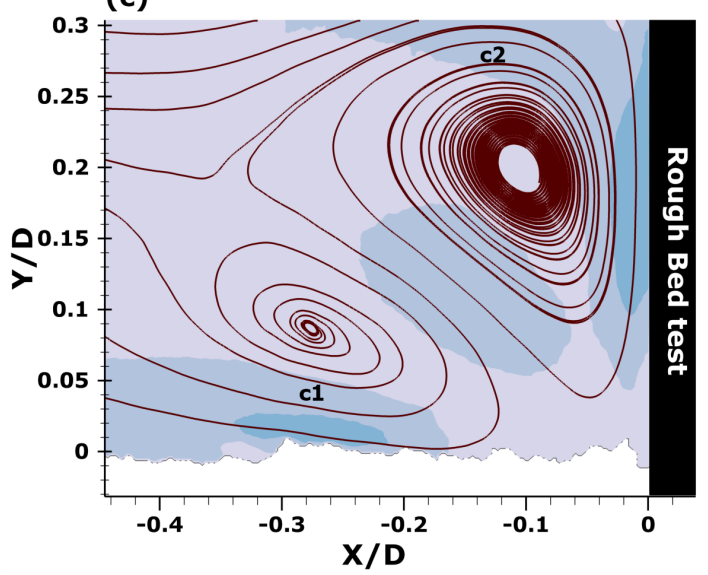

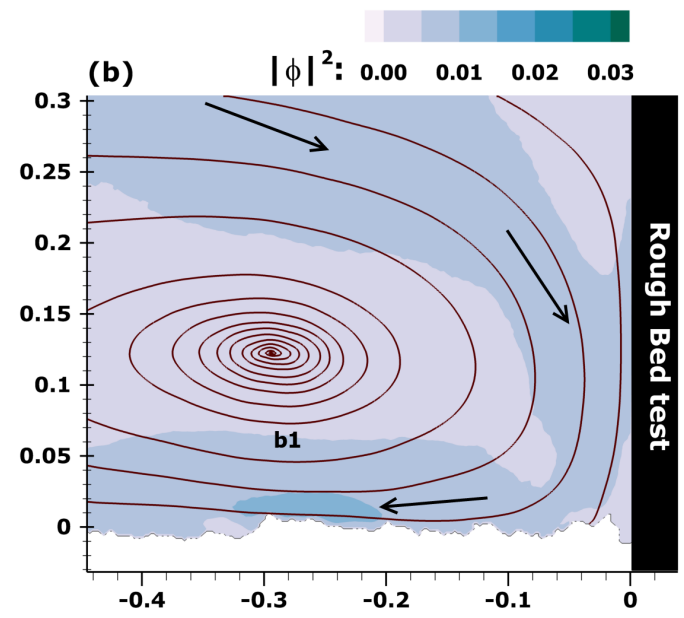

(d)

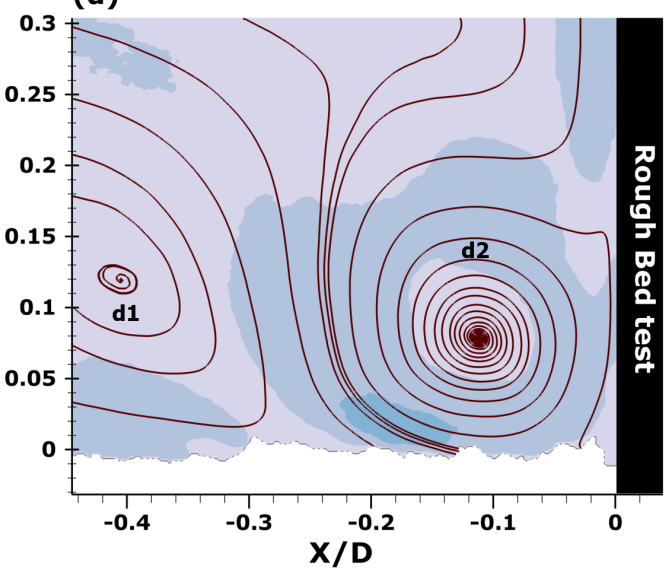

Fig. 12 Most energetic POD modes (pseudostreamlines) for the RB test: (a) first, (b) second, (c) third, and (d) fourth. The flood contour maps represent the Euclidean norm of the decomposition for the streamwise and wall-normal velocity signals

periodic flow components. This finding confirms the aperiodic behavior of the HVS at the particular levels of Re. The convergence of modal energy was qualitatively and quantitatively similar for both cases, alluding to the minor impact that the smaller flow scales (introduced by the presence of roughness) have on the effectiveness on POD.

The results and conclusions of this work apply to the junction flow parameters and roughness geometry characterizing the specific experimental setups under consideration. Any effort for generalization should consider the limitations of the study, such as the use of different Particle Image Velocimetry systems and degree of development of the incoming flow. Upon consideration of these facts, the findings presented here could be used to enhance the performance of generic models for junction flows based on theoretical arguments or numerical analysis. Benefits could also arise on the design of engineering applications, especially those characterized by irregular surface topographies (bridge pier scour, aerodynamics of damaged wing-fuselage junctions).
Acknowledgements We thank Professor P. P. Vlachos and Dr. S. Raben for their help and insights regarding the application of Particle Image Velocimetry in turbulent flows. National Science Foundation (EAR 0738759) and the Research Office of the U.S. Army Corps of Engineers (ARO 53512-EV) funded this project. Nikolaos Apsilidis acknowledges the generous support he received throughout the course of this study from: Academy of Athens (Argyropoulos fellowship), Gerondelis Foundation, Geological Society of America, and Sigma Xi: The Scientific Research Society.

\section{A Appendix}

The comparison between two experimental configurations for junction flows is the backbone of this research project. The two setups shared similarities, but had also differences. As discussed previously, the major discrepancy that could potentially cast doubts on the validity of the results is with respect to the sampling rates of the PIV systems. According to what has been reported in junction flow literature (Agui and Andreopoulos 1992, Devenport and Simpson 1990, Kirkil and Constantinescu 2009), the low sampling rate of SPIV $(7.5 \mathrm{~Hz})$ should still suffice to resolve the behavior of the large-scale flow structures comprising the 
(a)

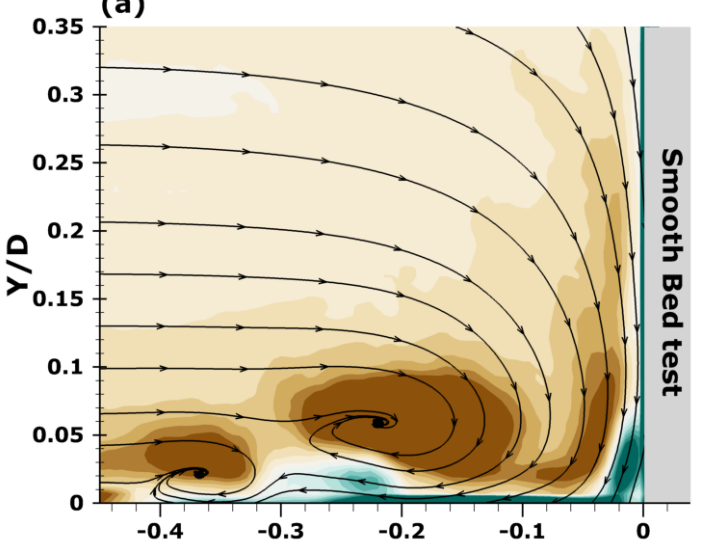

(c)

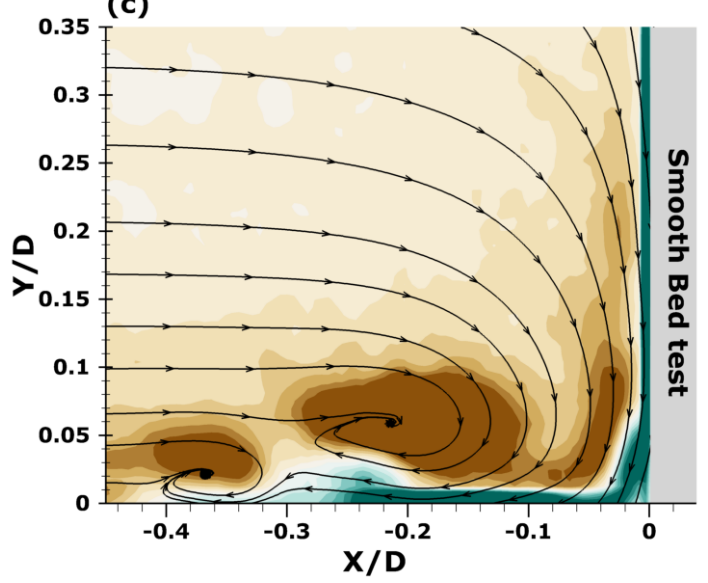

(b)

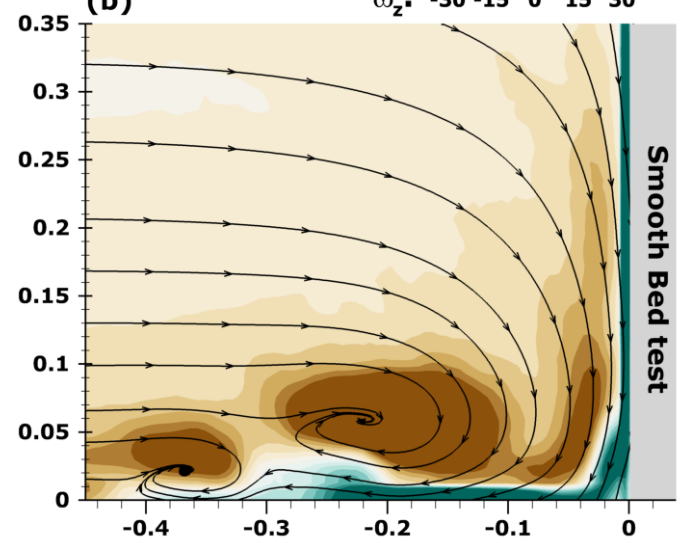

(d)

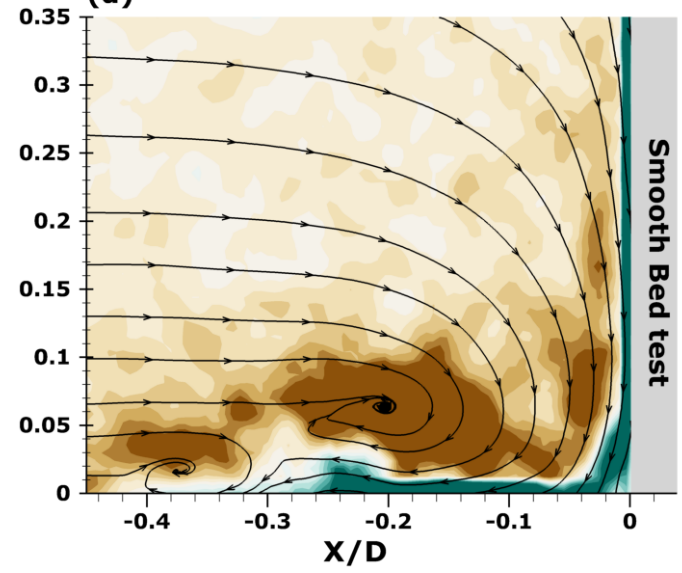

Fig. 13 Time-averaged streamlines and spanwise vorticity resolved from measurements with sampling frequencies of: (a) $1,000 \mathrm{~Hz}$, (b) $250 \mathrm{~Hz}$, (c) $50 \mathrm{~Hz}$, and (d) $7.5 \mathrm{~Hz}$

turbulent horseshoe vortex. Nevertheless, the nearly two orders of magnitude difference compared to the sampling rate of the TRPIV system $(1,000 \mathrm{~Hz})$ calls for further investigation. Towards this direction, downsampling on the TRPIV data was performed. Figure 13 illustrates the effects of reduced sampling frequency on the time-averaged streamlines and vorticity field. The operation was extended to the intermediate frequencies of $250 \mathrm{~Hz}$ and $50 \mathrm{~Hz}$ for completeness. It is shown that the basic features of the HVS topology remain essentially the same. Reasonable agreement is found with respect to the vorticity magnitudes. Namely, the same structures are resolved in every subfigure. As expected, the fidelity of the spatial resolution degrades somewhat with the reduction of the sampling rate (maps appear more "noisy"), but only to a negligible degree considering the scope of this work. Similar analyses were also performed for turbulence kinetic energy and intensity, but are not presented here to preserve the economy of writing space. These results revealed the same mild effect of the lower sampling rate on the calculated turbulence quantities near the cores of PV and SV. It is, finally, worth mentioning that 2,250 measurements were used to produce Figures $2 b, 3 b, 4 b$ and $5 b$, whereas the numbers of measurements used for Figures 13b, 13c and 13d were respectively 800, 160 and 24.

\section{References}

1. Adrian RJ, Westerweel J (2011) Particle Image Velocimetry. Cambridge University Press, New York

2. Agui JH, Andreopoulos J (1992) Experimental investigation of a three-dimensional boundary layer flow in the vicinity of an upright wall mounted cylinder. J Fluids Engng 114:566-576

3. Ahmed F, Rajaratnam N (1998) Flow around bridge piers. J Hydraul Engng 124:288-300

4. Berkooz G, Holmes P, Lumley JL (1993) The proper orthogonal decomposition in the analysis of turbulent flows. Annu Rev Fluid Mech 25:539-575

5. Cruz A, David L, Pecheux J, Texier A (2005) Characterization by proper-orthogonal-decomposition of the passive controlled wake flow downstream of a half cylinder. Exp Fluids 39:730-742 doi: 10.1007/s00348-005-0006-8

6. Devenport WJ, Simpson RL (1990) Time-dependent and timeaveraged turbulence structure near the nose of a wing-body junction. J Fluid Mech 210:23-55

7. Doligalski TL, Smith CR, Walker JDA (1994) Vortex interactions with walls. Annu Rev Fluid Mech 26:573-616

8. Eckstein A, Charonko J, Vlachos PP (2008) Phase correlation processing for DPIV measurements. Exp Fluids 45:485-500 
This is the peer reviewed version of the following article: Apsilidis, N., Diplas, P., Dancey, C.L. et al. Exp Fluids (2016) 57: 12. https://doi.org/10.1007/s00348-015-2098-0, which has been published in final form at link.springer.com. This article may be used for non-commercial purposes in accordance with Springer Terms and Conditions for Use of Self-Archived Versions.

9. Eckstein A, Vlachos PP (2009) Digital particle image velocimetry (DPIV) robust phase correlation. Meas Sci Technol 20:055401 doi: $10.1088 / 0957-0233 / 20 / 5 / 055401$

10. El Hassan M, Meslem A (2010) Time-resolved stereoscopic particle image velocimetry investigation of the entrainment in the near field of circular and daisy-shaped orifice jets. Phys Fluids 22:035107 doi: 10.1063/1.3358465

11. Escauriaza C, Sotiropoulos F (2011) Reynolds number effects on the coherent dynamics of the turbulent horseshoe vortex system. Flow Turbul Combust 86:231-262 doi: 10.1007/s10494-010-9315-y

12. Gand F, Deck S, Brunet V, Sagaut P (2010) Flow dynamics past a simplified wing body junction. Phys Fluids 22:115111

13. Holmes $P$ (2012) Turbulence, coherent structures, dynamical systems and symmetry. Cambridge University Press, Cambridge, UK

14. Honkanen M, Nobach H (2005) Background extraction from double-frame PIV images. Exp Fluids 38:348-362 doi: $10.1007 / \mathrm{s} 00348-004-0916-\mathrm{x}$

15. Jolliffe IT (2002) Principal component analysis. Springer, New York

16. Kirkil G, Constantinescu G (2009) Nature of flow and turbulence structure around an in-stream vertical plate in a shallow channel and the implications for sediment erosion. Water Resour Res 45:W06412 doi: 10.1029/2008wr007363

17. Kirkil G, Constantinescu G, Ettema R (2006) Investigation of the velocity and pressure fluctuations distributions inside the turbulent horseshoe vortex system around a circular bridge pier. In: Ferreira RML, Alves E, Leal J and Cardoso AH (eds) River Flow 2006. Balkema, Lisbon, pp. 709-718

18. Koken M, Constantinescu G (2009) An investigation of the dynamics of coherent structures in a turbulent channel flow with a vertical sidewall obstruction. Phys Fluids 21:085104 doi: 10.1063/1.3207859

19. Lumley JL (1967) The structure of inhomogeneous turbulent flows. In: Yaglom AM and Tatarski VI (eds) Atmospheric turbulence and radio propagation. Nauka, pp. 166-178

20. Nguyen TD, Wells JC, Mokhasi P, Rempfer D (2010) Proper orthogonal decomposition-based estimations of the flow field from particle image velocimetry wall-gradient measurements in the backwardfacing step flow. Meas Sci Technol 21:115406

21. Paik J, Escauriaza C, Sotiropoulos F (2007) On the bimodal dynamics of the turbulent horseshoe vortex system in a wing-body junction. Phys Fluids 19:115104 doi: 10.1063/1.2716813

22. Peridier VJ, Smith FT, Walker JDA (1991) Vortex-induced boundary-layer separation. Part 1. The unsteady limit problem $\mathrm{Re} \rightarrow \infty$. J Fluid Mech 232:99-131

23. Peridier VJ, Smith FT, Walker JDA (1991) Vortex-induced boundary-layer separation. Part 2. Unsteady interacting boundarylayer theory. J Fluid Mech 232:133-165

24. Praisner TJ, Smith CR (2006) The dynamics of the horseshoe vortex and associated endwall heat transfer - Part I: Temporal behavior. J Turbomach 128:747-754 doi: 10.1115/1.2185676

25. Praisner TJ, Smith CR (2006) The dynamics of the horseshoe vortex and associated endwall heat transfer - Part II: Time-mean results. J Turbomach 128:755-762 doi: 10.1115/1.2185677

26. Raffel M, Willert CE, Wereley ST, Kompenhans J (2007) Particle Image Velocimetry-A practical guide. Springer

27. Roulund A, Sumer BM, Fredsoe J, Michelsen J (2005) Numerical and experimental investigation of flow and scour around a circular pile. J Fluid Mech 534:351-401 doi: 10.1017/S002112005004507

28. Sabatino DR, Smith CR (2009) Boundary layer influence on the unsteady horseshoe vortex flow and surface heat transfer. J Turbomach 131:011015 doi: 10.1115/1.2813001

29. Sagaut $P$ (2006) Large eddy simulation for incompressible flows : An introduction. Springer-Verlag, Berlin

30. Scarano F (2002) Iterative image deformation methods in PIV. Meas Sci Technol 13:R1-R19

31. Shavit U, Lowe R, Steinbuck J (2007) Intensity capping: a simple method to improve cross-correlation PIV results. Exp Fluids 42:225240 doi: 10.1007/s00348-006-0233-7
32. Simpson RL (2001) Junction flows. Annu Rev Fluid Mech 33:415443

33. Sirovich L (1987) Turbulence and the dynamics of coherent structures: 1. Coherent structures. Quarterly of Applied Mathematics 45:561-571

34. Spalart PR (2009) Detached-Eddy Simulation. Annu Rev Fluid Mech 41:181-202 doi: 10.1146/annurev.fluid.010908.165130

35. Tsikata JM, Tachie MF (2013) Adverse pressure gradient turbulent flows over rough walls. Int J Heat Fluid Flow 39:127-145 doi: http://dx.doi.org/10.1016/j.ijheatfluidflow.2012.11.001

36. Van Dommelen LL, Shen SF (1980) The spontaneous generation of the singularity in a separating laminar boundary layer. J Comput Phys 38:125-140

37. Westerweel J (1997) Fundamentals of digital particle image velocimetry. Meas Sci Technol 8:1379-1392

38. Westerweel J, Elsinga GE, Adrian RJ (2013) Particle image velocimetry for complex and turbulent flows. Annu Rev Fluid Mech 45:409-436 doi:10.1146/annurev-fluid-120710-101204

39. Westerweel J, Scarano F (2005) Universal outlier detection for PIV data. Exp Fluids 39:1096-1100 doi: 10.1007/s00348-005-0016-6 